\title{
An Alternative Tibial Osteotomy Technique
}

\author{
Christopher lobst
}

\begin{abstract}
Introduction: The anterior approach to a tibial osteotomy commonly employs a small incision and multiple drill holes followed by completion with an osteotome. Potential complications are damage to the anterior tibial periosteum and posterior neurovascular structures.

Materials and Methods: We describe an alternative method using two small incisions, which avoids elevation of the anterior tibial periosteum and directs the osteotome away from posterior neurovascular structures.

Results: This technique has been successfully performed on 15 consecutive tibias, with no neurovascular complications or skin healing issues. Conclusion: This tibial osteotomy technique can be performed quickly and safely using a minimally invasive approach as an alternative to the traditional anterior approach.

Keywords: Anterior approach, Tibial osteotomy, Tibial periosteum.

Strategies in Trauma and Limb Reconstruction (2020): 10.5005/jp-journals-10080-1458
\end{abstract}

\section{INTRODUCTION}

Tibial osteotomies using the multiple drill holes and the osteotome method are performed through a small incision using an anterior approach. There are three potential drawbacks to this method: ${ }^{1}$

- latrogenic damage to the anterior tibial periosteum is often unavoidable in order to gain appropriate access to the bone. This periosteal stripping may lead to an anterior defect in the subsequent regenerate bone.

- The anterior approach compels the surgeon to direct the osteotome in an anterior-to-posterior direction. The posterior neurovascular structures are at risk to injury if the osteotome is inadvertently advanced too far posteriorly.

- To visualise the depth of osteotome penetration while using the anterior approach, the C-arm must be placed in the lateral position. This orientation of the C-arm can be inefficient and awkward for the surgeon.

An alternative tibial osteotomy technique has been developed that will help to alleviate these concerns.

\section{Materials and Methods}

The alternative tibial osteotomy technique uses two small incisions (Fig. 1).

- A 5-mm incision is made over the anterior crest of the tibia. A drill bit is then directed anterior to posterior parallel to the lateral cortex of the tibia to create a bicortical drill hole. The drill is then withdrawn from the posterior cortex and readvanced slightly medial to the first exit hole creating a second posterior hole. This is repeated two more times until the final hole is through the posteromedial corner of the tibia. This pattern of drilling uses one anterior entry hole to produce a total of four posterior cortex holes (Fig. 2). The size of the drill bit is at the surgeon's discretion but a 4.8-mm drill bit for adult sized tibias and a 3.8-mm drill bit for paediatric sized tibias was used by the author.

- A 5-10-mm incision in the skin at the same level is made at the posteromedial edge of the tibia. The drill is directed straight medial to lateral parallel to the posterior cortex to create a
Department of Orthopedic Surgery, Nationwide Children's Hospital, Columbus, Ohio, USA

Corresponding Author: Christopher lobst, Department of Orthopedic Surgery, Nationwide Children's Hospital, Columbus, Ohio, USA, Phone: +1 614-722-3371, e-mail: christopher.iobst@nationwidechildrens.org

How to cite this article: lobst C. An Alternative Tibial Osteotomy Technique. Strategies Trauma Limb Reconstr 2020;15(1):47-49.

Source of support: Nil

Conflict of interest: None

bicortical drill hole (Fig. 3). A second bicortical drill path is made parallel to the first hole in a medial-to-lateral direction. Using the second medial entry hole, the drill is angled anteriorly and a hole is made in the anterolateral corner of the tibia. Finally, the
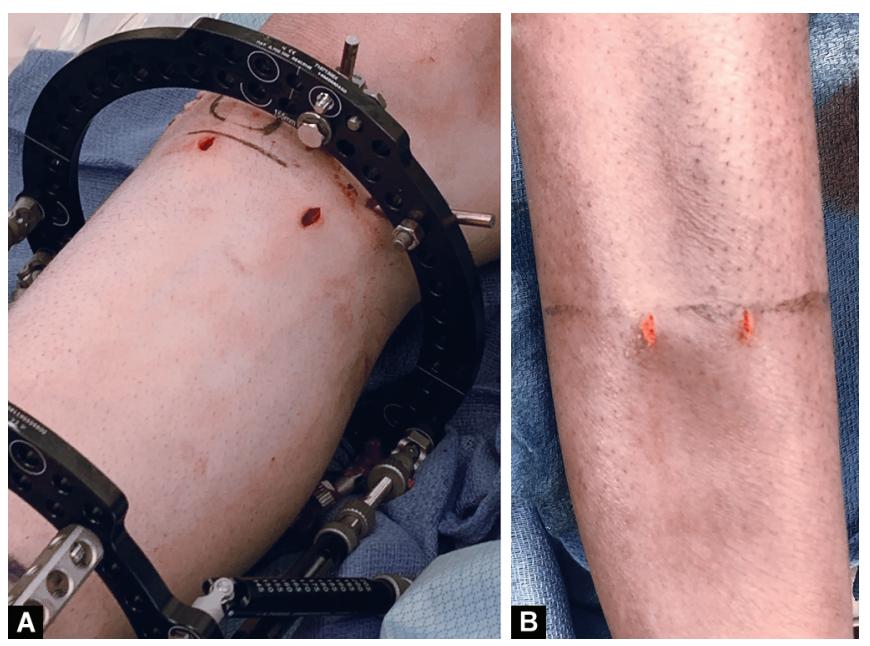

Figs $1 \mathrm{~A}$ and $\mathrm{B}$ : (A) Clinical photographs of skin incisions for a proximal tibial metaphyseal osteotomy using this alternative technique; (B) Clinical photographs of skin incisions for a tibial diaphyseal osteotomy using this alternative technique

(c) The Author(s). 2020 Open Access This article is distributed under the terms of the Creative Commons Attribution 4.0 International License (https://creativecommons. org/licenses/by-nc/4.0/), which permits unrestricted use, distribution, and non-commercial reproduction in any medium, provided you give appropriate credit to the original author(s) and the source, provide a link to the Creative Commons license, and indicate if changes were made. The Creative Commons Public Domain Dedication waiver (http://creativecommons.org/publicdomain/zero/1.0/) applies to the data made available in this article, unless otherwise stated. 


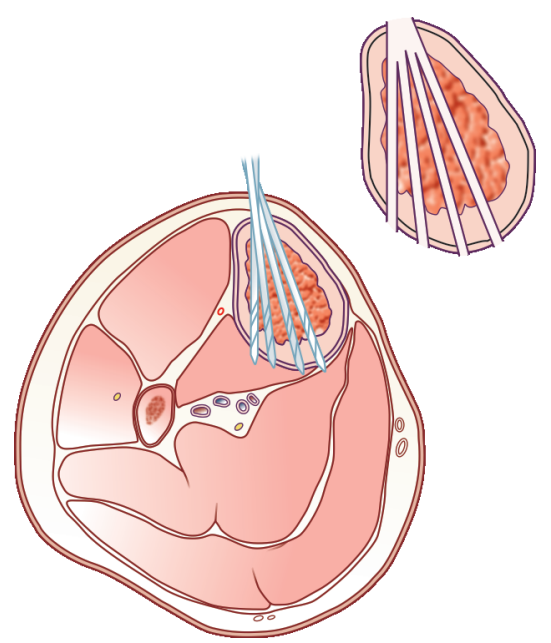

Fig. 2: Illustration of the anterior-to-posterior drill hole pattern through a 5-mm skin incision

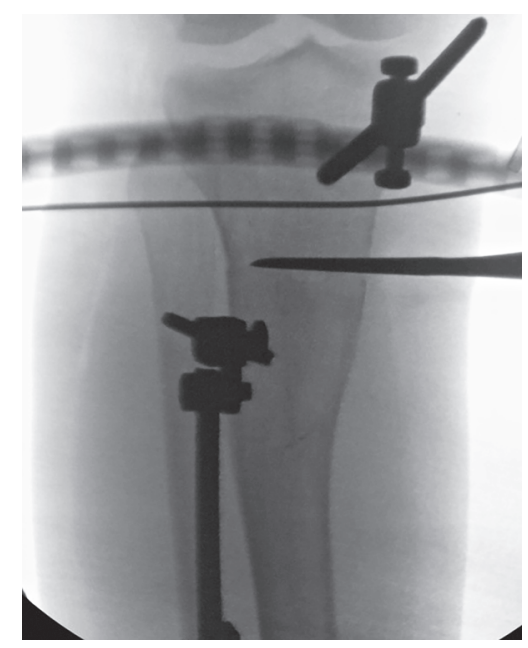

Fig. 4: Fluoroscopic intraoperative image demonstrating that the osteotome is passed from medial to lateral to complete the osteotomy

skin can be retracted posteriorly and a unicortical hole can be made in the posteromedial tibia.

- With the limb elevated on towels, the osteotome is used to pass from medial to lateral through the medial incision (Fig. 4). The medial cortex of the tibia is also cut, aiming from posterior to anterior. At this point, a twist of the inserted osteotome should be sufficient to complete the osteotomy. While the size of the osteotome is ultimately determined by the size of the skin incision, the author used a $6-\mathrm{mm}$ (1/4 inch) osteotome.

\section{Results}

This osteotomy technique has been successfully performed on 15 consecutive tibias. The same technique was used in the tibia regardless of osteotomy location (proximal metaphysis, diaphysis, and distal metaphysis). The osteotomy created a smooth, transverse bone cut without spikes or irregularities in each case. There were no neurovascular complications. There were no skin-healing issues. The tibia fully healed without noticeable regenerated bone defects in each case. In each case, the surgical time to complete the osteotomy using this technique was $<5$ minutes.

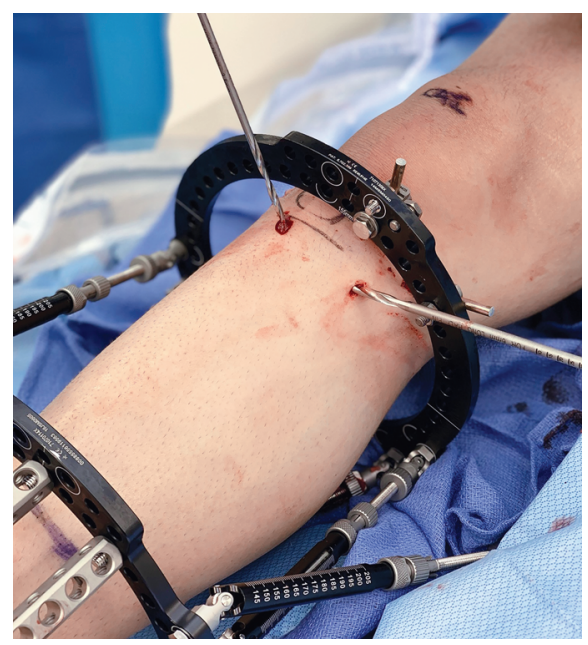

Fig. 3: Clinical photograph of opposing drill bits demonstrating the anterior-to-posterior path and the medial-to-lateral path of the drill
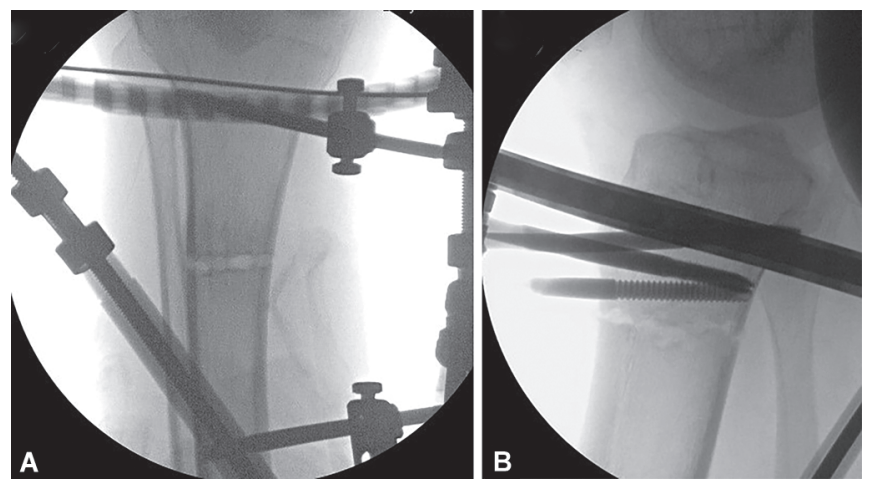

Figs $5 \mathrm{~A}$ and $\mathrm{B}$ : Intraoperative fluoroscopic images demonstrating the circumferential drill hole pattern created using this technique

\section{Discussion}

This alternative tibial osteotomy technique has been performed on 15 consecutive patients, both in the metaphysis and the diaphysis. There are several advantages of this method: (1) The technique avoids elevating the anterior periosteum. Stripping of the anterior periosteum, even in minimally invasive approaches, can lead to poor anterior bone formation and delayed healing; (2) The technique is potentially safer than the traditional anterior approach because it directs the osteotome away from the neurovascular structures. In this alternative method, the osteotome is advanced from medial to lateral and/or from posterior to anterior rather which prevents an inadvertent plunge of the osteotome blade away from the posterior tibial artery and nerve; (3) The intraoperative fluoroscopy machine does not need to be placed in the lateral orientation to visualise the osteotome path.

The C-arm can be angled out of the way of the surgeon and kept in the normal anterior-to-posterior orientation throughout the osteotomy process. This avoids the difficulty of working around the machine or the extra time required to repeatedly reposition the machine or the limb to check the osteotome position; and (4) The creation of circumferential drill holes appears to decrease the tendency to cause spikes or large irregularities at the osteotomy site that commonly occurred with drill holes only from the anterior approach (Figs 5 and 6). 


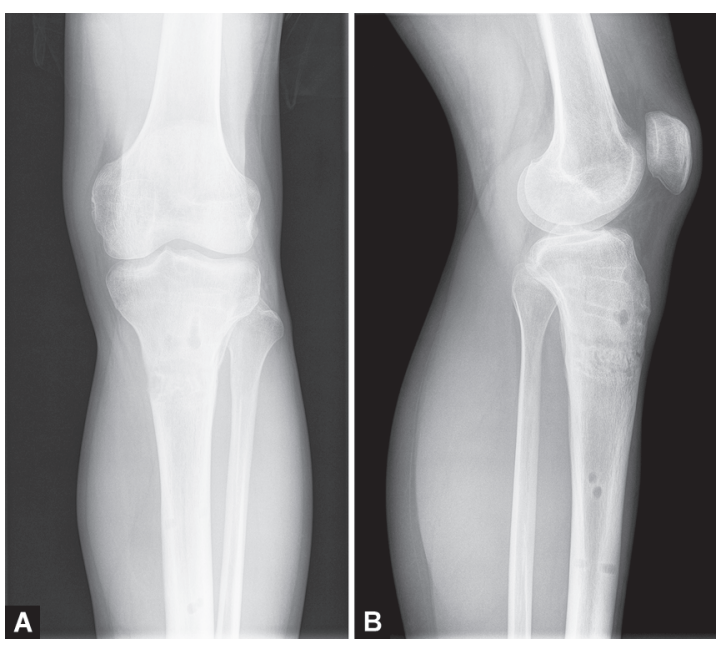

In conclusion, this tibial osteotomy technique can be performed quickly and safely using a minimally invasive approach as an alternative to the traditional anterior approach.

\section{Availability of Data and Material}

Data are available upon reasonable request to the author.

\section{Reference}

1. Paley D. Hardware and osteotomy considerations. In: Paley D, ed. Principals of Deformity Correction. Berlin; 2002. pp. 291-410.

Figs $6 \mathrm{~A}$ and $\mathrm{B}$ : Clinical radiographs demonstrating the healed regenerate bone of a patient after deformity correction and $2.5 \mathrm{~cm}$ lengthening using the described alternative tibial osteotomy technique 